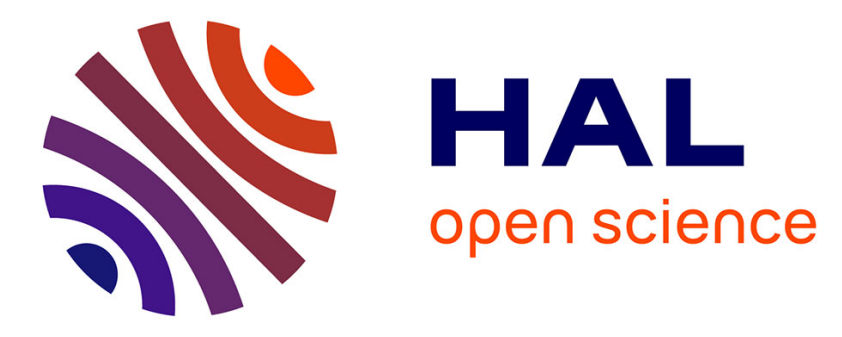

\title{
Stress transmission in cemented bidisperse granular materials
}

\author{
K. Heinze, Xavier Frank, Valerie V. Lullien-Pellerin, Matthieu George, \\ Farhang Radjai, Jean-Yves Delenne
}

\section{- To cite this version:}

K. Heinze, Xavier Frank, Valerie V. Lullien-Pellerin, Matthieu George, Farhang Radjai, et al.. Stress transmission in cemented bidisperse granular materials. Physical Review E , 2020, 101 (5), 10.1103/PhysRevE.101.052901 . hal-02573271

\section{HAL Id: hal-02573271 \\ https://hal.inrae.fr/hal-02573271}

Submitted on 4 Sep 2020

HAL is a multi-disciplinary open access archive for the deposit and dissemination of scientific research documents, whether they are published or not. The documents may come from teaching and research institutions in France or abroad, or from public or private research centers.
L'archive ouverte pluridisciplinaire $\mathbf{H A L}$, est destinée au dépôt et à la diffusion de documents scientifiques de niveau recherche, publiés ou non, émanant des établissements d'enseignement et de recherche français ou étrangers, des laboratoires publics ou privés. 


\title{
Stress transmission in cemented bidisperse granular materials
}

\author{
K. Heinze $\odot,{ }^{1,2, *}$ X. Frank,,${ }^{1}$ V. Lullien-Pellerin $\odot,{ }^{1}$ M. George, ${ }^{2}$ F. Radjai, ${ }^{3}$ and J.-Y. Delenne $\odot^{1, \dagger}$ \\ ${ }^{1}$ IATE, Université de Montpellier, INRA, CIRAD, Montpellier SupAgro, Montpellier, France \\ ${ }^{2}$ L2C, Université de Montpellier, CNRS, Montpellier, France \\ ${ }^{3}$ LMGC, Université de Montpellier, CNRS, Montpellier, France
}

(Received 25 October 2019; revised manuscript received 20 February 2020; accepted 8 April 2020; published 4 May 2020)

\begin{abstract}
We analyze stress distributions in a two-dimensional bidisperse cemented granular packing for a broad range of the values of particle-size ratio, the volumes of large and small particles, and the amount of cementing matrix. In such textured porous materials, the stress concentration, which controls the fracture and fragmentation of the material under tensile loading or in grinding processes, reflects not only the porosity but also the contact network of the particle phase and the resulting stress chains. By means of peridynamic simulations under tensile loading, we show how both the texture and stress distribution depend on size ratio, volume ratio, and the amount of the cementing matrix. In particular, the volume fraction of the class of small particles plays a key role in homogenizing stresses across the system by reducing porosity. Interestingly, the texture controls not only the porosity but also the distribution of pores inside the system with its statistical variability, found to be strongly correlated with the homogeneity of stresses inside the large particles. The most homogeneous stress distribution occurs for the largest size ratio and largest volume fraction of small particles, corresponding to the lowest pore size dispersion and the cushioning effect of small particles and its similar role to the binding matrix for stress redistribution across the packing. At higher porosity, the tensile stresses above the mean stress fall off exponentially in all phases with an exponent that strongly depends on the texture. The exponential part broadens with decreasing matrix volume fraction and particle-size ratio. These correlations reveal the strong interplay between size polydispersity and the cohesive action of the binding matrix for stress distribution, which is significant for the behavior of textured materials in grinding operations.
\end{abstract}

DOI: 10.1103/PhysRevE.101.052901

\section{INTRODUCTION}

Cemented granular materials (CGM) consist of particles that form a dense granular backbone consolidated by a cementing phase. This class of materials includes some biomaterials such as wheat endosperm [1-3], sedimentary rocks [4,5], and building materials such as concrete [6]. Their elastic and failure properties are therefore of broad interest and raise at the same time fundamental questions as to the combined effects of microtexture and cementing matrix on the mechanical behaior.

The microtexture of a CGM can be described in terms of the spatial organization of the particles and binding phase, including possible empty spaces (voids) in the matrix. A key feature of the microtexture is the granular contact network, which leads to a strongly inhomogeneous distribution of stresses, underlying the elastic properties and failure behavior. Among various system parameters, the particle-size distribution (PSD) [7-11], particle shapes [12,13], and porosity and pore shapes $[2,3,14-16]$ have been at the focus of several recent investigations, many of them based on simulations by the discrete element method (DEM). Often, the effect of each material parameter (particle shape, particle sizes, phase

\footnotetext{
*karsta.heinze@umontpellier.fr

†jean-yves.delenne@inra.fr
}

volume fractions, etc.) is considered independently of the effects of other parameters. However, parameters such as particle-size ratio, porosity, and cementing matrix volume are linked to one another, and their influence on the mechanical behavior can not be considered separately.

In this paper, we present a parametric study of the combined effects of particle-size distribution and cementing phase volume fraction on stress transmission in a CGM. The stress transmission features are crucial as an intermediate information between the microstructure and the mechanical behavior in response to external loading. In other words, the elastic properties and failure of a CGM are related to the distribution of stresses in the particle and matrix phases as well at the contact zones between particles. The elastic properties and failure CGMs will be at the focus of a forth-coming paper. Here, we focus on stress transmission by considering a two-dimensional CGM consisting of a dense packing of spherical disks, whose interstitial space is partially filled with a cementing matrix. The bidisperse-size distribution of the spherical particles is described in terms of two parameters: the size ratio and the volume ratio of large particles to small particles. These parameters are varied independently within a broad range of values.

With this broad array of samples, we construct a large number of microtextures within the parameter range. In each sample, a binding matrix is added to the particle assembly. The amount of matrix is varied between the two extremes of 


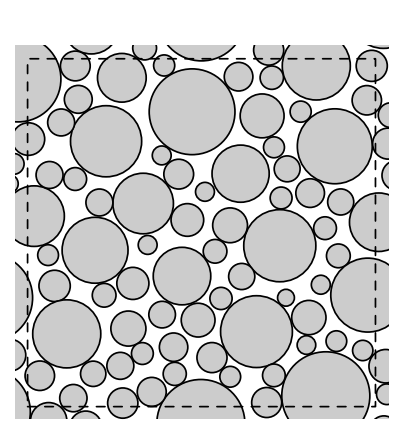

(a)

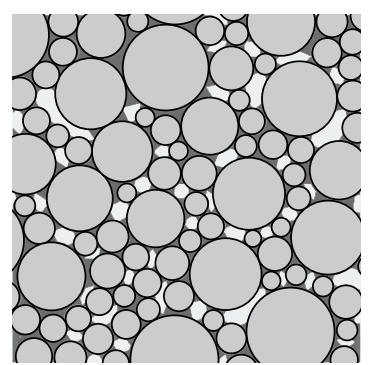

(c)

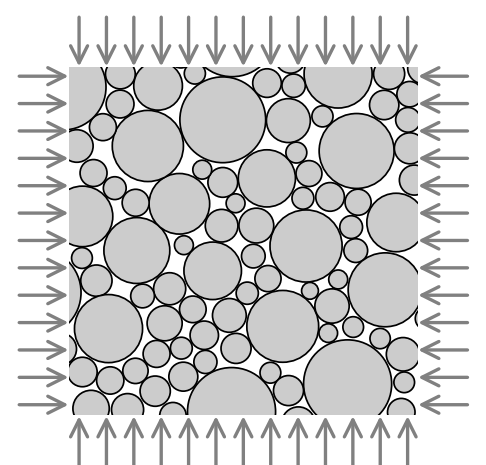

(b)

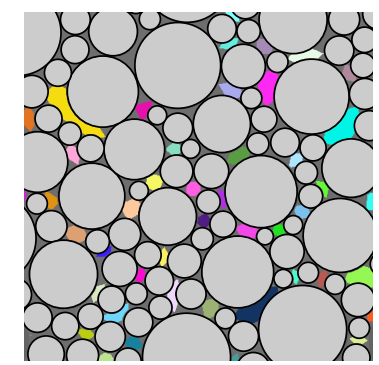

(d)
FIG. 1. Successive steps of sample preparation procedure. A zoom into a sample after (a) FPDS, (b) DEM compression, and (c) addition of cementing matrix. (d) The voids in the partially cemented sample and their sizes were identified by a floodfill routine.

very low and completely filling the interstitial space. For the simulations, we use the peridynamic method, which makes it possible to simulate both the matrix (as a continuous phase) and the particles. We characterize the microtextures and stress distributions under simple tensile deformation.

In the following, we first describe the numerical procedures, including sample preparation and the peridynamic method in Sec. II. In Sec. III, we investigate the general

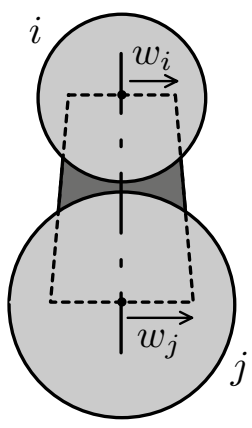

(a)

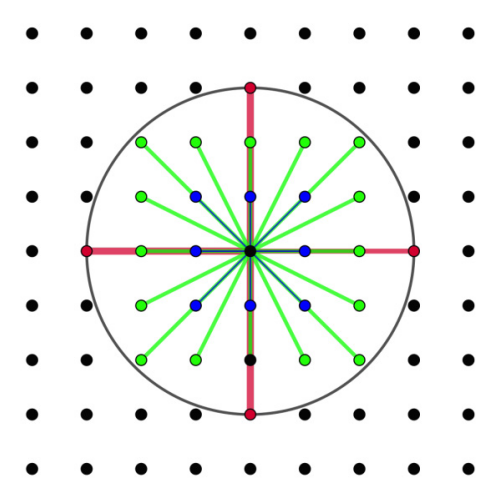

(b)
FIG. 2. (a) Trapezoidal bridge between two disks (Only the dark gray part is considered in the simulations). (b) The bonds of a central node (in black) with the nodes located inside a horizon of $h=3 \delta_{x}$ are shown, in different colors for a better visualization. A horizon of $h=3 \delta_{x}$ was used throughout the study.

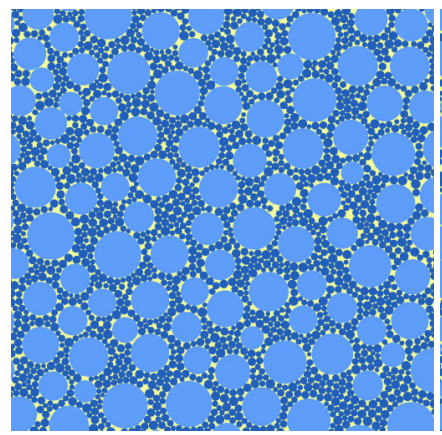

(a)

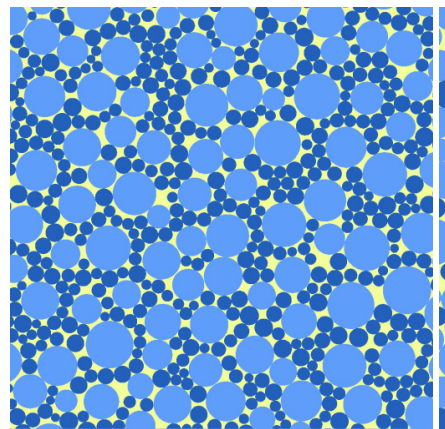

(c)

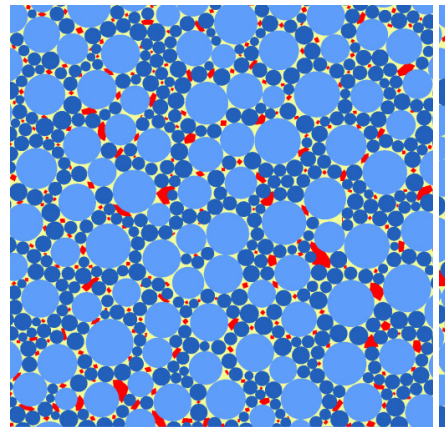

(e)

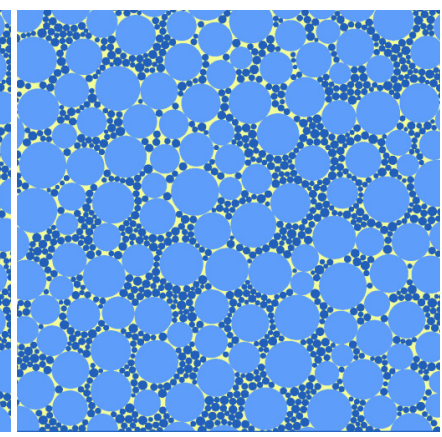

(b)

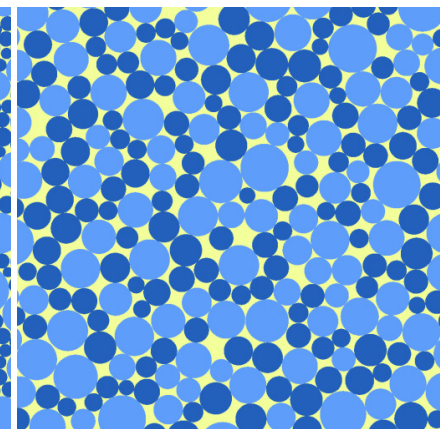

(d)

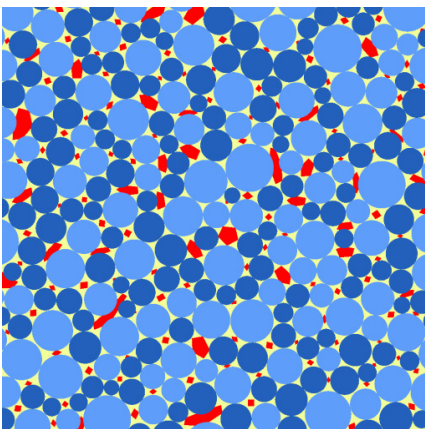

(f)
FIG. 3. Snapshots of samples for different values of the size and volume ratios $\gamma$ and $\theta$, respectively, of particles and void fraction $\rho_{v}$ : (a) $\gamma=5.00, \theta=1.4, \rho_{v}=0$; (b) $\gamma=5.00, \theta=2.7, \rho_{v}=$ 0 ; (c) $\gamma=2.50, \theta=1.4, \rho_{v}=0$; (d) $\gamma=1.43, \theta=1.4, \rho_{v}=0$; (e) $\gamma=2.50, \theta=1.4, \rho_{v}=0.15$; (f) $\gamma=1.43, \theta=1.4, \rho_{v}=0.18$. Large particles are colored in light blue, small particles in dark blue, the cement in yellow and the voids in red.

characteristics of the microtextures. Then, in Sec. IV, we investigate the stress distributions. We conclude with the most salient results of this work and its possible extensions.

\section{NUMERICAL PROCEDURES}

The samples are composed of disklike particles, a binding matrix, and voids of their volume fractions $\rho_{p}, \rho_{m}$, and $\rho_{v}$, respectively. This section describes the sample generation procedure and the basics of the bond-based peridynamic method used for the calculation of stresses under tensile loading.

\section{A. Particle-size distribution}

The particle phase consists of disks with a bimodal-size distribution characterized by volume fractions $\rho_{L}$ for large 


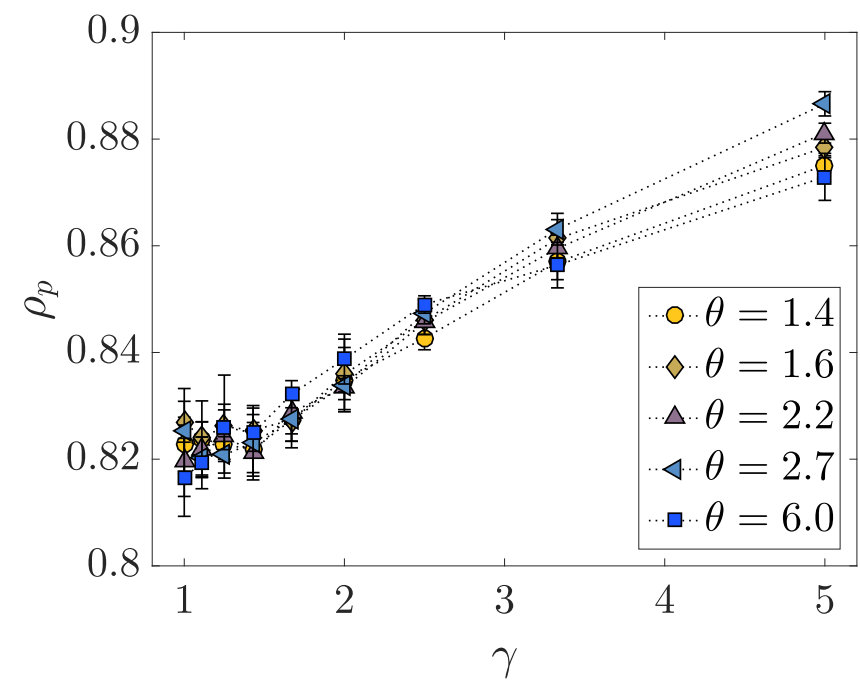

(a)

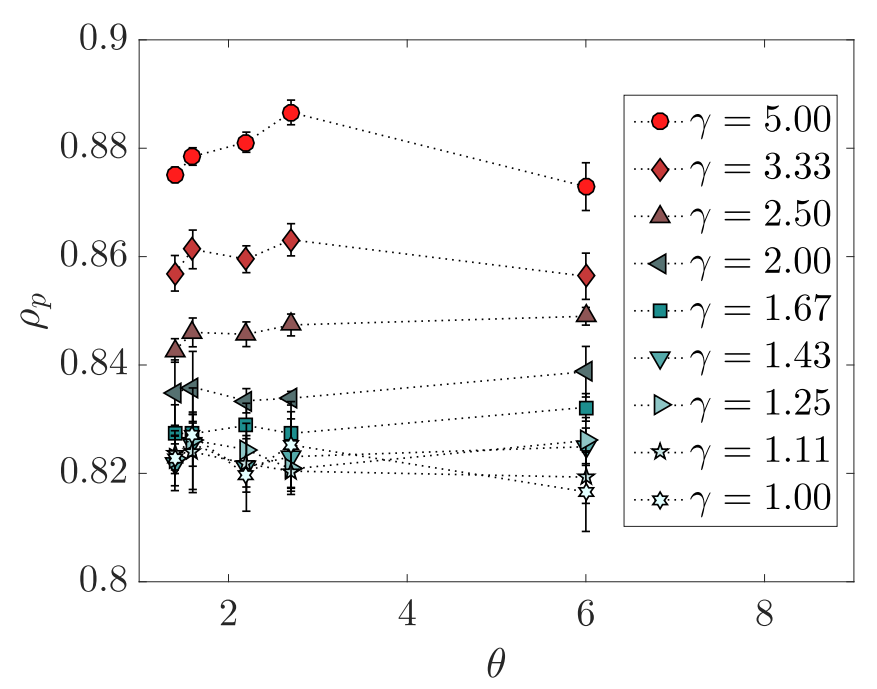

(b)

FIG. 4. The evolution of particle volume fraction $\rho_{p}$ with (a) $\gamma$ and (b) $\theta$. The data are the average values over 5 samples with their standard deviations represented as error bars.

disks (of mean radius $\overline{r_{L}}$ ) and $\rho_{S}$ for small disks (of mean radius $\overline{r_{S}}$ ). With these notations, the total particle volume fraction is $\rho_{p}=\rho_{L}+\rho_{S}$. To reduce local ordering of the particles [12], a normal distribution with $\sigma_{r_{L}}=0.25 \overline{r_{L}}$ or $\sigma_{r_{S}}=0.25 \overline{r_{S}}$ is introduced on the sizes of each of the two particle types. Due to the finite resolution of the mesh used for the peridynamics simulations, we only kept the particles in the range of $\left[\overline{r_{S}}-1.5 \sigma_{r_{S}}, \overline{r_{L}}+1.5 \sigma_{r_{L}}\right]$. With these cutoff values the smallest disks were meshed by about 10 nodes along their diameters.

The particle-size distribution depends on two parameters: the ratio between the mean radii of large and small disks,

$$
\gamma=\overline{r_{L}} / \overline{r_{S}}
$$

and the ratio of volume fractions of large and small particles,

$$
\theta=\rho_{L} / \rho_{S}
$$

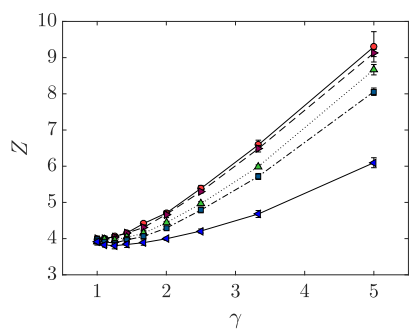

(a)

$\bullet-\theta=1.4 \rightarrow \theta=1.6 \backsim \theta=2.2 \nleftarrow \theta=2.7 \nleftarrow \theta=6.0$

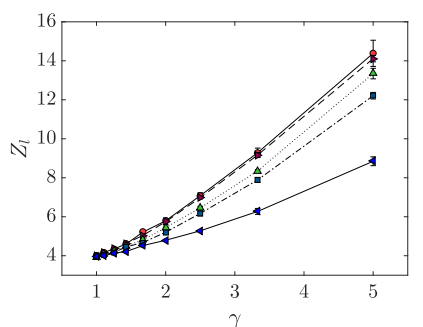

(b)

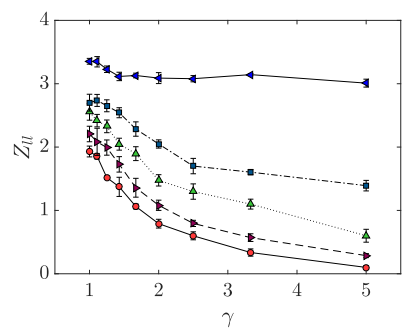

(d)

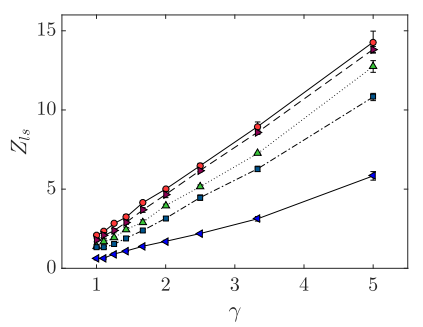

(f)

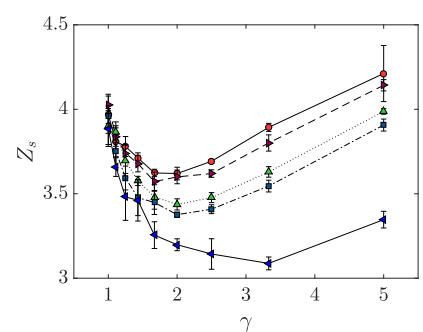

(c)

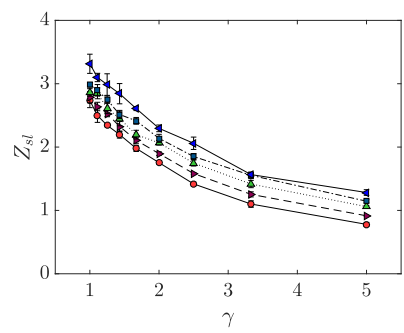

(e)

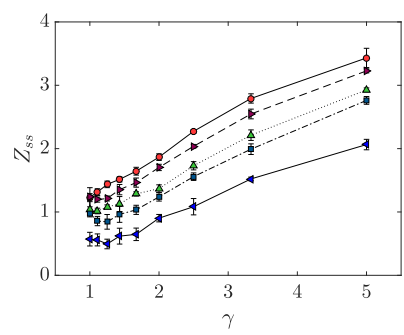

(g)
FIG. 5. Partial coordination numbers as a function of volume ratio $\gamma$ for different values of size ratio $\theta$. See Eq. (9) and text for the definitions.

The variation of $\gamma$ was controlled by keeping $\overline{r_{L}}$ constant while $\overline{r_{S}}$ was varied from $\overline{r_{S}}=0.2 \overline{r_{L}}$ to $\overline{r_{S}}=\overline{r_{L}}$. The resulting values of $\gamma$ were $\{5.00,3.33,2.50,2.00,1.67,1.43,1.25$, $1.11,1.00\}$. Five different values of $\theta$ were considered: $\theta=$ $[1.4(0.1), 1.6(0.1), 2.2(0.2), 2.7(0.3), 6.0(0.9)]$. In this way, 45 different combinations of $\gamma$ and $\theta$ were obtained. For each combination, five statistically independent samples were created. Note that we did not consider lower values of $\theta$, since, as we shall see below, the main results of this work depend marginally on $\theta$ in this limit. The configurations considered in this work are thus such that the small particles fill the pore space between large particles. Lower values of $\theta$ represent configurations in which the large particles are embedded in a packing of small particles. 


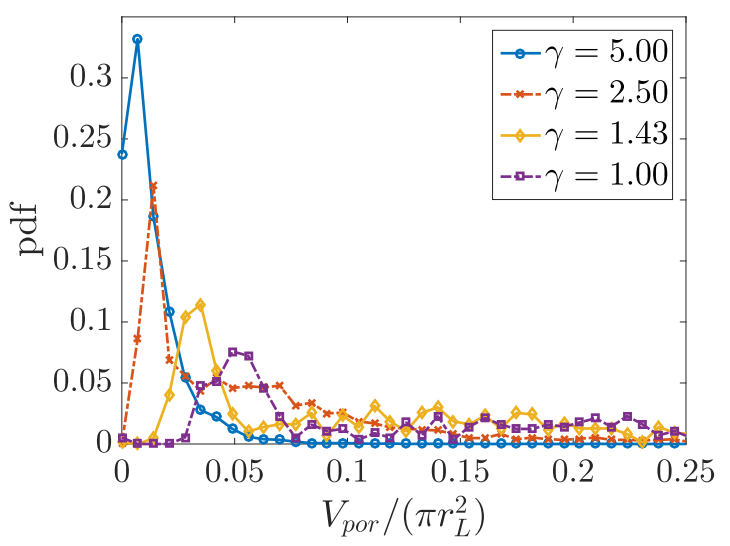

(a)

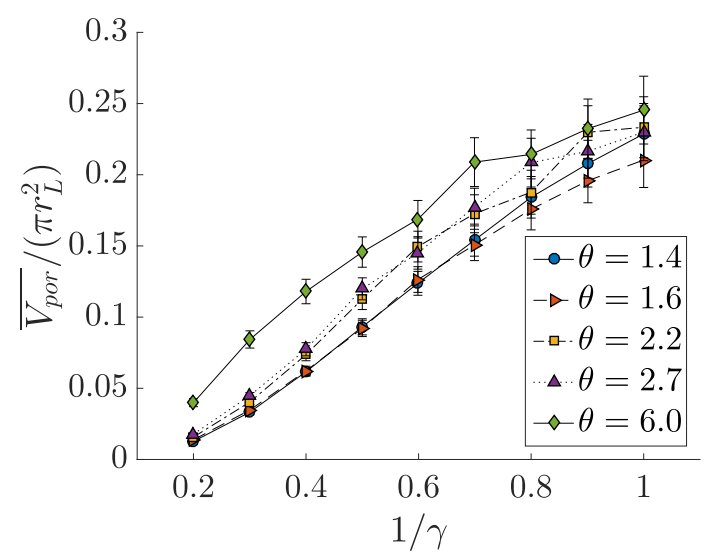

(b)

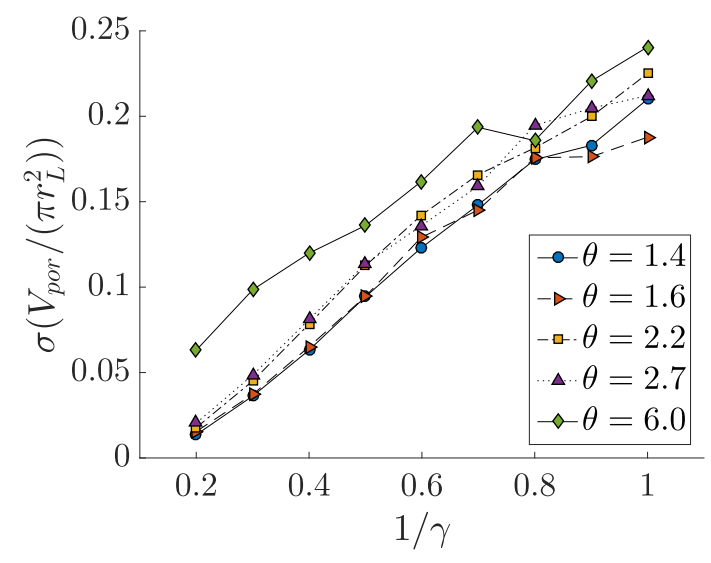

(c)

FIG. 6. Single-pore volume (inter-grain spaces) for $\theta=1.4$ : (a) pdfs of normalized single-pore volumes for selected values of $\gamma$, (b) average normalized single-pore volume, and (c) standard deviation of normalized single-pore volume versus small particle size $r_{s} \propto 1 / \gamma$. The points represent the average value over five samples. Error bars in (b) show the standard deviation around the average data point.

\section{B. Sample construction}

Sample construction proceeds by first introducing large particles and then adding smaller particles in the remaining free space. A square element of area $A_{\mathrm{box}}=900 \pi{\overline{r_{L}}}^{2}$ was filled with large disks of randomly attributed radius $r_{L}$ from the normal distribution characterized by the mean radius $\overline{r_{L}}$ and standard deviation $\sigma_{r_{L}}$, using fast Poisson disk sampling (FPDS) [17]. FPDS consists of randomly placing a high number of preliminary disks, each with a random radius from within the normal distribution of radii, around a randomly picked existing disk. The preliminary disks are consecutively tested for overlap with existing disks and if no such overlap is detected, are permanently added to the sample. These steps are repeated until after a reasonable number of iterations no more disks could be added. For more details, see the Appendix. We applied a two-step FPDS, consisting of first placing large disks and in the second step placing the small disks. A minimum distance $d_{\min }(\gamma, \theta) \geqslant 0$ between large disks was used to allow for sufficient free space for the subsequent placement of small disks, allowing us to achieve the desired values of $\theta$. Once the square element was filled with large disks, the small disks were placed using the same FPDS method, but without a minimum distance requirement.

This two-step FPDS assembly leads to a nearly dense packing of disks [Fig. 1(a)]. To further increase the density, we applied biaxial compression to the samples by means of discrete element simulations. The samples were compressed with a ratio of normal contact stiffness $k_{n}$ to confining pressure $k_{n} / P=10^{4}$, which is large enough to verify the "hard particle" approximation, and a Coulomb coefficient of friction $\mu=0.3$ [Fig. 1(b)].

The matrix was added to the compacted samples by the procedure described by Topin et al. [2]. Trapezoidal bridges were created between all pairs of disks (disk $i$ and disk $j$ ) separated by a distance below or equal to a small length $\ell$ [Fig. 2(a)]. The width of the two bridge bases $w_{i}$ and $w_{j}$ are a fraction $\eta$ of the radius $r$ of the corresponding disks: $w_{i}=\eta \times$ $r_{i}$ and $w_{j}=\eta \times r_{j}$. The matrix volume fraction was varied by increasing $\eta$ in 5 steps of 0.2 from 0.2 to 1.0. The resulting overall porosity ranges from $\rho_{v}=0.0$ to 0.97 .

Square regions of fixed size were cropped out of the center of the box and meshed onto a two-dimensional rectilinear grid of 1024 by 1024 nodes. Each node was attributed to a phase (particle, matrix, void). The phase volume fractions were determined by counting the number of nodes within each phase. The floodfill routine $[18,19]$ was used to identify the voids and interstitial spaces. Figure 1(c) shows an example of a partially filled sample. As a result of space discretization and cropping, the final values of $\theta$ vary slightly between samples.

\section{Bond-based peridynamic method}

Peridynamics is based on the integral formulation of the equations of dynamics instead of partial differential equations [20]. The equation of motion of a material point located at a point $\mathbf{x}$ is given by

$$
\operatorname{Pü}(\mathbf{x}, t)=\int_{\mathcal{H}_{x}} \mathbf{f}\left(\mathbf{u}\left(\mathbf{x}^{\prime}, t\right)-\mathbf{u}(\mathbf{x}, t), \mathbf{x}-\mathbf{x}^{\prime}\right) d V_{x^{\prime}}+\mathbf{b}(\mathbf{x}, t),
$$

where $\mathrm{P}$ is mass density, $\mathcal{H}_{x}$ is a neighbourhood around $\mathbf{x}$, $\mathbf{u}$ is the displacement field, and $\mathbf{b}$ is the body force. The neighborhood is generally defined as a sphere of radius $h$, called horizon, centered on $\mathbf{x}$. $\mathbf{f}$ is a pairwise force between 


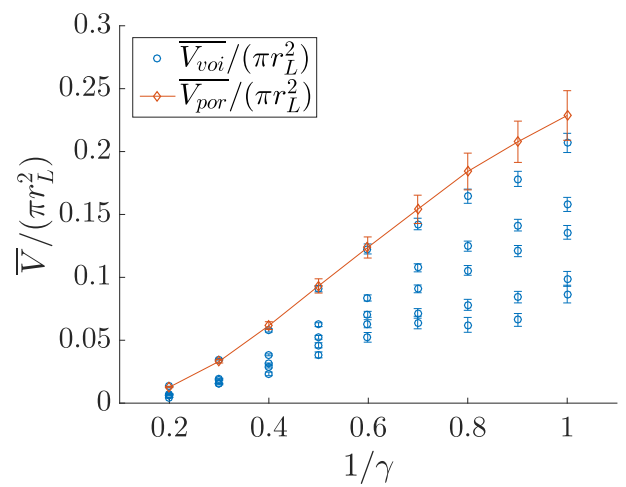

(a)

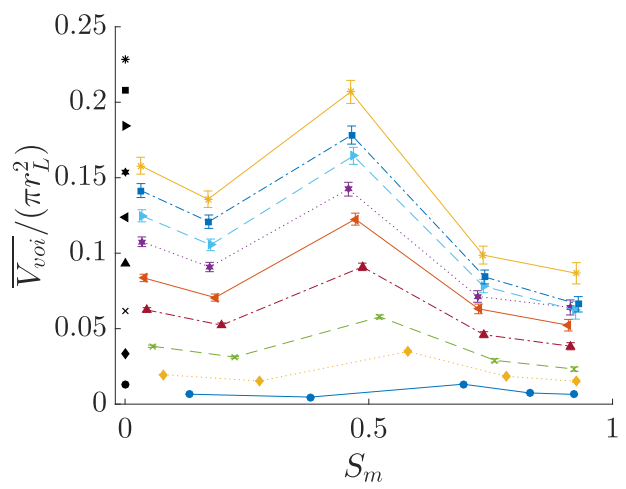

(b)

$\bullet \gamma=5.00 \bullet \gamma=3.33-\gamma=2.50-\uparrow=2.00 \longleftarrow \gamma=1.67 \cdots \gamma=1.43 \rightarrow \gamma=1.25-\gamma=1.11 * \gamma=1.00$

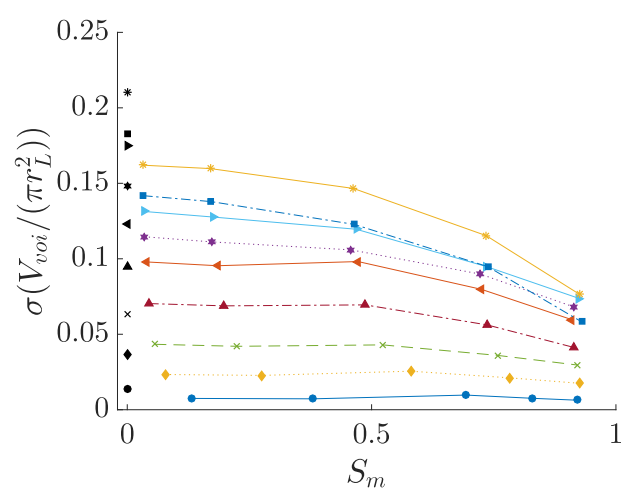

(c)

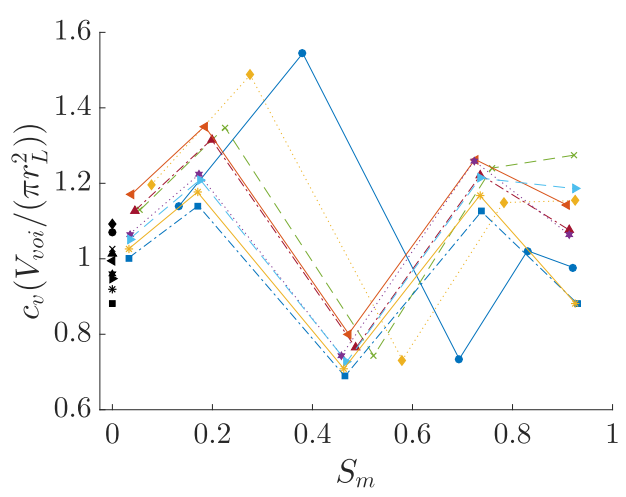

(d)

FIG. 7. For $\theta=1.4$ : (a) The blue points show the average normalized single-void volume (with standard error of the mean) for samples with different amounts of cementing matrix versus small particle size $r_{s} \propto 1 / \gamma$. For comparison, the average normalized single-pore volume for each particle-size configuration is shown in orange. (b) The mean normalized single-void volume versus matrix saturation $S_{m}$. (c) The standard deviation of the normalized single-void volume versus matrix saturation and (d) the coefficient of variation of the normalized single-void volume versus matrix saturation $S_{m}$. All points are the average values taken over 5 independent samples. The black symbols in (b), (c), and (d) show the average normalized values for pores in the corresponding samples.

$\mathbf{x}^{\prime}$ and $\mathbf{x}$. This force is a function of the distance $\xi=\mathbf{x}-\mathbf{x}^{\prime}$ and the relative displacement $\eta=\mathbf{u}\left(\mathbf{x}^{\prime}, t\right)-\mathbf{u}(\mathbf{x}, t)$ [21]. The interaction is represented by a bond and its elongation $s$ is defined by

$$
s(\xi, \eta)=\frac{\|\xi+\eta\|-\|\xi\|}{\|\eta\|} .
$$

The micromodulus $c$ and Young modulus $E$ in two dimensions are related via the following equation [22]:

$$
c_{\lambda_{x} \lambda_{x^{\prime}}}=\frac{6 E}{\pi h^{3}(1-v)},
$$

with Poisson ratio $v=1 / 3$. In multiphase media, the pairwise force $\mathbf{f}_{x x^{\prime}}$ depends on the materials forming the bond:

$$
\mathbf{f}_{x x^{\prime}}= \begin{cases}c_{\lambda_{x} \lambda_{x^{\prime}}} s(\xi, \eta) n & \|\xi\| \leqslant h \\ 0 & \|\xi\|>h\end{cases}
$$

where $\lambda(x)$ is the index of the phase to which belongs the point $x$ and $n=(\xi+\eta) /\|\xi+\eta\|$ is an orientated unitary vector.
The simulated domain is discretized into a 1024 by 1024 rectilinear grid of spatial resolution $\delta x$. Each material point $i$ of position $\mathbf{x}_{i}$ has a mass $m_{i}=(\delta x)^{2} \rho\left(\mathbf{x}_{i}\right)$. The system can be viewed as a mass-spring lattice in which each bond (linear spring) connects two points on the grid. The equation of motion Eq. (3) is discretized on the grid:

$$
m_{i} \ddot{\mathbf{u}}_{i}(t)=\sum_{\mathbf{x}_{j} \in \mathcal{H}\left(\mathbf{x}_{i}\right)} k_{\lambda_{i} \lambda_{j}} s\left(\boldsymbol{\xi}_{i j}, \boldsymbol{\eta}_{i j}\right) \frac{\boldsymbol{\xi}_{i j}+\boldsymbol{\eta}_{i j}}{\left\|\boldsymbol{\xi}_{i j}+\boldsymbol{\eta}_{i j}\right\|}+\mathbf{b}_{i}(t),
$$

where $\mathbf{u}_{i}(t)=\mathbf{u}\left(\mathbf{x}_{i}, t\right), \boldsymbol{\xi}_{i j}=\mathbf{x}_{j}-\mathbf{x}_{i}, \boldsymbol{\eta}_{i j}=\mathbf{u}_{j}-\mathbf{u}_{i}, \mathbf{b}_{i}(t)=$ $(\delta x)^{2} \mathbf{b}\left(\mathbf{x}_{i}, t\right), k=c(\delta x)^{4}, \lambda_{i}=\lambda\left(\mathbf{x}_{i}\right)$, and $\lambda_{j}=\lambda\left(\mathbf{x}_{j}\right)$.

Since no excluded-volume constraints are imposed on the points, the strain should be kept small enough to avoid overlapping between neighboring bonds and ensure a macroscopic linear behavior. The number of bonds interacting with each grid point depends on $h / \delta_{x}$. Figure 2(b) shows an example of bond connectivity for the horizon radius $h=3 \delta_{x}$ used in this study.

To compute the evolution of the system, we use an explicit time integration scheme with a viscous force $-v \dot{\mathbf{u}}_{i}(t)$ applied 


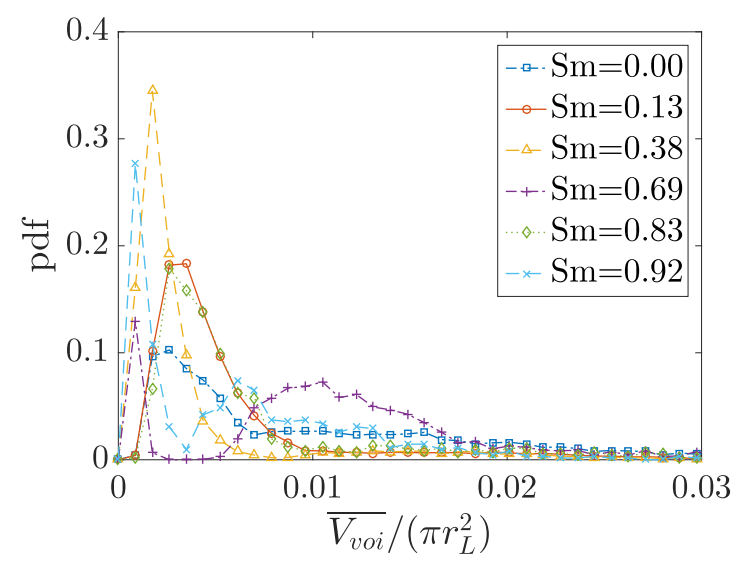

(a)

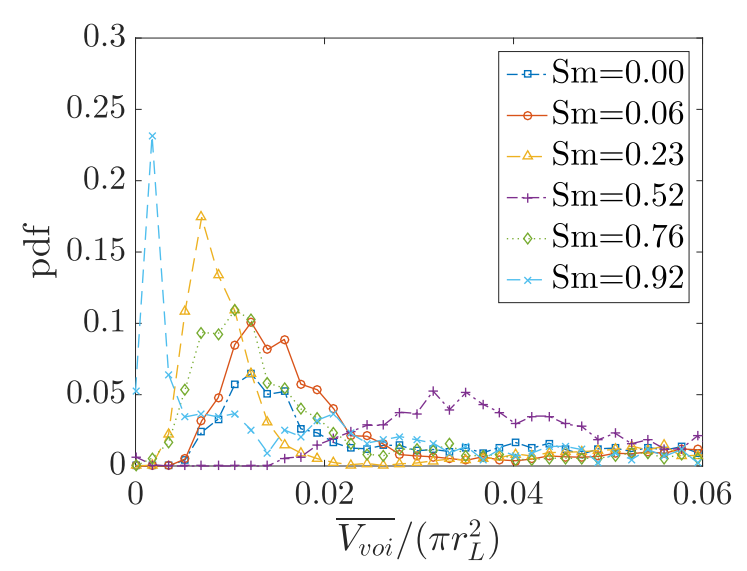

(b)

FIG. 8. The PDFs of single-void volumes in samples with $\theta=$ 1.4 for (a) $\gamma=5.00, \theta=1.4$ and (b) $\gamma=2.50, \theta=1.4$ and different values of matrix saturation $S_{m}$.

to each material point to damp elastic waves. The viscosity $v$ should be below the critical viscosity $\min \left\{\sqrt{2 m_{i} k}\right\}$ to avoid supercritical damping.

Finally, the stress tensor at each point $i$ is computed by summing all contributions of connected bonds using $\sigma_{i}=$ $\frac{1}{2 V_{i}} \sum_{j} f_{i j} \otimes \xi_{i j}$, where $f_{i j}=\mathbf{f}_{x x^{\prime}}$ and $V_{i}=\delta_{x}^{2}$.

\section{Mechanical parameters}

The bonds connecting two nodes belonging to the same particle are given the particle phase properties independently of particle sizes. In the same way, the bonds connecting two nodes both belonging to the matrix phase are given the matrix phase properties. The bonds connecting two nodes belonging to two different particles or connecting one particle node to one matrix node are classified as interface bonds with their specified properties. Hence, we have three types of bonds: particle-particle $p$, matrix-matrix $m$ and particle-matrix $p m$. Each bond type is given an elastic modulus $\left(E_{p}, E_{m}, E_{p m}\right)$ and a toughness $\left(K_{p}, K_{m}, K_{p m}\right)$, summarized in Table I. In this work, we assume that the $p m$ bonds and $m$ bonds have the same properties. This choice does not affect stress transmission. But it can be important for fracture, e.g. if the interface has a weaker strength compared to the particles and matrix.
TABLE I. The elasticity and toughness parameters for the three bond types.

\begin{tabular}{lcc}
\hline \hline Matrix & Particle & Interface \\
\hline$E_{m}$ & $E_{p}=4 E_{m}$ & $E_{i}=E_{m}$ \\
$K_{m}$ & $K_{p}=3.5 K_{m}$ & $K_{i}=K_{m}$ \\
\hline \hline
\end{tabular}

The values of these mechanical parameters will be in the same range as those used by Chichti et al. [3], who took into account the measured values of starch granules and protein matrix in wheat endosperm by atomic force microscopy [23,24]. Hence, the results of this work apply directly to the wheat endosperm microstructure although most conclusions are general.

The samples were subjected to quasi-static uniaxial tension in the $y$ direction. The lower boundary was fixed, the side boundaries were mobile, while the top line of nodes was displaced at a constant speed. The tensile deformation was pursued for a few steps. All samples show linear elastic behavior.

\section{GRANULAR MICROTEXTURE}

Several examples of packings with different particle-size distributions (PSD) are displayed in Fig. 3. We clearly see that the particle arrangements have qualitative differences according to the values of size and volume ratio. When the binding matrix is added in different proportions to these arrangements, the resulting microstructure gets even more complex.

The particle volume fraction $\rho_{p}$ varies in the range from 0.82 and 0.89. Its value is displayed in Fig. 4 as a function of $\gamma$ for different values of $\theta$, and conversely. $\rho_{p}$ increases rapidly with $\gamma$ except at low values of the latter. This observation is in agreement with a number of studies that investigated the packing density of two-dimensional (2D) bidisperse disks [25-28] and three-dimensional (3D) binary assemblies of spheres [29]. According to these studies, the densest packing is expected when large particles constitute about $70 \%$ of the particle fraction, corresponding to $\theta \approx 2.3$. For this value of $\theta$, the amount of small particles would be just high enough to fill in most of the available space between large particles. In our case, the value for the highest observed particle volume fraction is slightly higher at $\theta=2.7$. This is caused by the normal distribution of particle sizes around the two defined radii of the two types of particles. The real peak of $\rho_{p}$ could, however, also lie in between 2.7 and 6.0 since that region was not sampled. From the peak toward higher values of $\theta$, the decrease in the number and volume of small particles leads to more and more unfilled spaces between the large particles [27]. In our samples, this influence of $\theta$ was clearly observed only for $\gamma=5.00$. For $\gamma<5, \theta$ has little effect on $\rho_{p}$.

The coordination number gives the average number of contacts that a particle has with its neighbors. This number provides an important structural information for the distribution of stresses via the particle contact network. Two particles are counted as being in contact if their distance is less or equal to the size of the horizon, because this is the condition for bond formation between the nodes of particles. The total and 


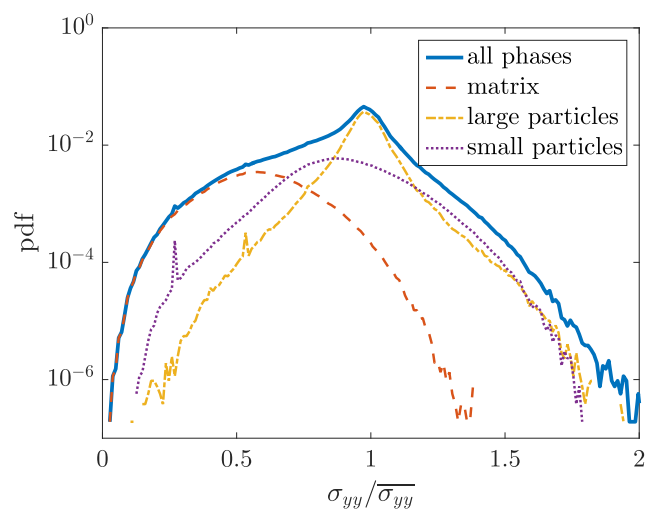

(a)

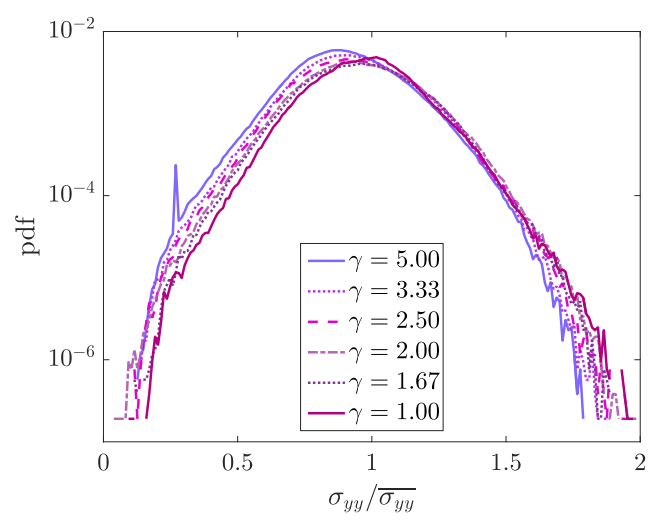

(c)

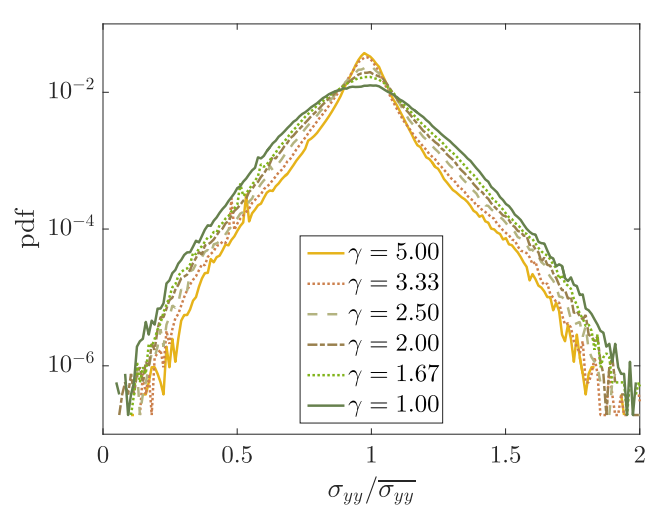

(b)

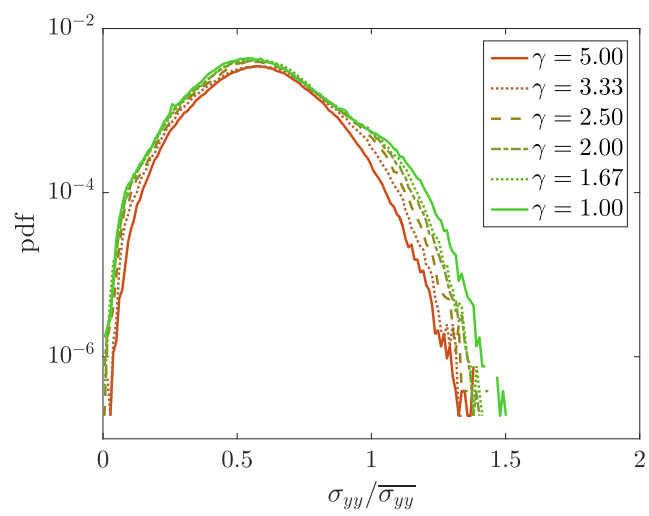

(d)

FIG. 9. Normalized vertical stress distributions $\sigma_{y y} / \overline{\sigma_{y y}}$ in full samples: (a) in different phases for $\gamma=5.00$ and $\theta=1.4$, (b) in large particles and, (c) in small particles, and (d) in matrix.

partial coordination numbers [26] are defined as follows:

$$
\begin{aligned}
Z_{\lambda \lambda^{\prime}} & =\sum_{\lambda_{i}=1}^{n_{\lambda}} \sum_{\lambda_{j}^{\prime}=1}^{n_{\lambda}^{\prime}} \delta / n_{\lambda}, \\
\delta & = \begin{cases}1 & \sqrt{\left(x_{\lambda_{i}}-x_{\lambda_{j}^{\prime}}\right)^{2}+\left(y_{\lambda_{i}}-y_{\lambda_{j}^{\prime}}\right)^{2}} \leqslant h, \\
0 & \sqrt{\left(x_{\lambda_{i}}-x_{\lambda_{j}^{\prime}}\right)^{2}+\left(y_{\lambda_{i}}-y_{\lambda_{j}^{\prime}}\right)^{2}}>h, \\
0 & \lambda_{i}=\lambda_{j}^{\prime} .\end{cases}
\end{aligned}
$$

Partial coordination numbers are denoted: $Z_{l l}$ for contacts between large particles, $Z_{l s}$ for the large particle contacts with small particles, $Z_{l}=Z_{l l}+Z_{l s}$ for all contacts of large particles, $Z_{s l}$ for small particle contacts with large particles, $Z_{s s}$ for contacts between small particles and $Z_{s}=Z_{s l}+Z_{s s}$ for all contacts of small particles. The overall coordination is $Z=\left(Z_{l}+Z_{s}\right) / 2$.

The global coordination number $Z$ increases with $\gamma$ as shown in Fig. 5(a), but the partial coordination numbers of large and small particles show quite distinct behaviours. The partial coordination number of large particles $Z_{l}$ is increasing constantly with $\gamma$ [Fig. 5(b)] for all values of $\theta$. This increase is caused by a strong increase of the number of small particles around large particles $\left[Z_{l s}\right.$, Fig. 5(f)], which largely compensates the decrease in the number of large-large particle contacts $\left[Z_{l l}\right.$, Fig. 5(d)]. This increase of $Z_{l s}$ is almost linear and most likely caused by the fact that the increase in $\gamma$ goes along with an increase in the absolute number of small particles. The decrease of the number of large-large contacts $Z_{l l}$ with $\gamma$ is barely affecting the overall number $Z_{l}$, but highlights the shrinking of the large-large particle contact network. For $\theta \leqslant 2.2$ and $\gamma>2, Z_{l l}$ can drop to values below 1 , showing that in these packings large particles are mostly isolated from one another.

The partial coordination numbers of small particles also show two opposite effects. As $Z_{l s}, Z_{s s}$ increases with $\gamma$ due to the increase of the absolute number of small particles [Fig. 5(g)]. However, as the size of small particles decreases, they form patches rather than single small particles located between large particles. This increasing structuring of small particles into clusters causes $Z_{s l}$ to decrease [Fig. 5(e)], as small particles are more likely to be surrounded by other small particles than by large particles. In contrast to $Z_{l}$, both effects are of the same order, and they result together in an initial decrease in $Z_{s}$ with increasing $\gamma$, followed by an increase when a critical value of $\gamma$, depending on $\theta$, is reached.

The overall variation of $Z_{S}$ [Fig. $\left.5(\mathrm{c})\right]$ is quite small $(3.1 \leqslant$ $Z_{s} \leqslant 4.2$ ) compared to the variation of $Z_{l}$ in the range $4 \leqslant$ $Z_{l} \leqslant 14$. For all values of $\theta$, it first declines with $\gamma$ before increasing again for larger values of $\gamma$. This unmonotonic variation reflects the variations of $Z_{s l}$ and $Z_{s s}$. At low values of $\gamma, Z_{s l}$ declines rapidly whereas $Z_{s s}$ increases slowly, leading to a small decrease of $Z_{s}$. At high values of $\gamma, Z_{s l}$ declines less rapidly while $Z_{s s}$ continues to increase at the same rate, leading to an increase of $Z_{s}$. 


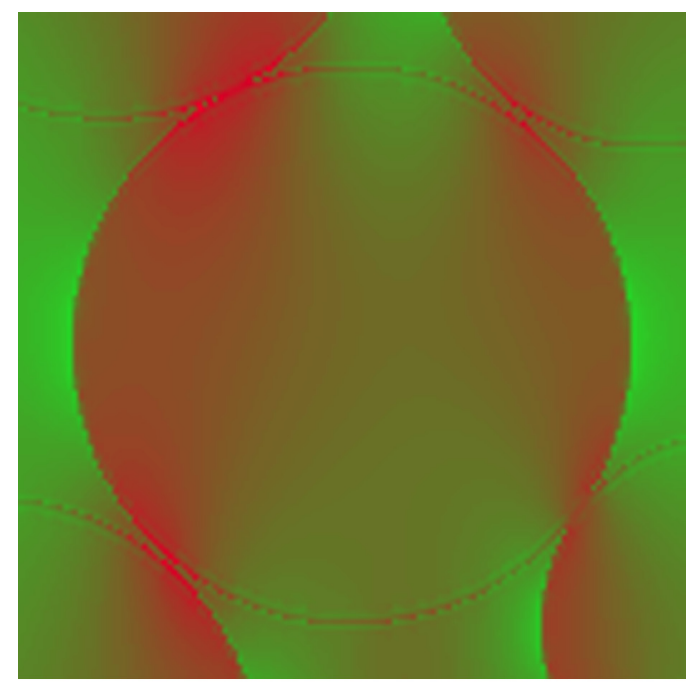

(a)

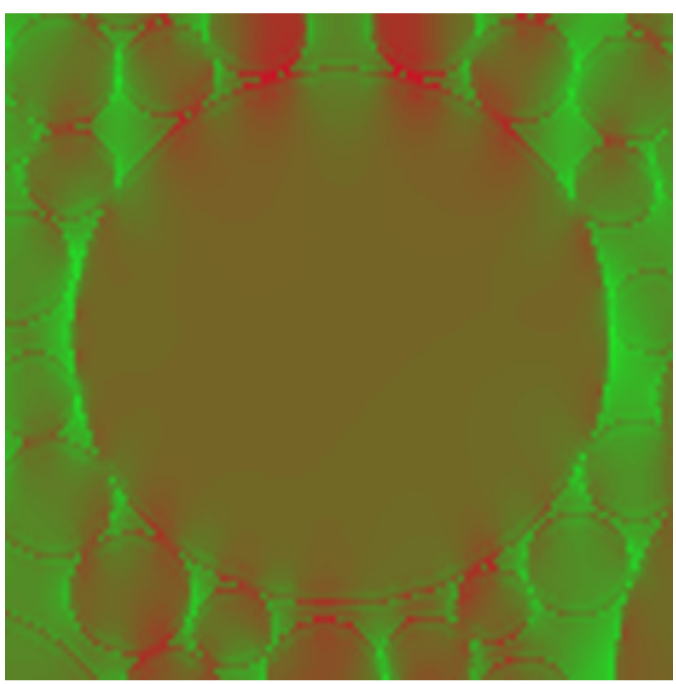

(b)

FIG. 10. Zoom on one large particle, showing the distribution of stresses from their low values (green) to high values (red) for (a) $\gamma=$ 3.33 and (b) $\gamma=5.00$.

In the following, the term "pore volume" describes the interstitial space (all the space not occupied by particles): $1-$ $\rho_{p}$. A "single" pore is an interstitial space enclosed by a closed chain of contiguous particles; see Fig. 1(d). Additionally, the term "void volume" describes empty space: $1-\rho_{p}-\rho_{m}$. A "single" void is an empty space enclosed by matrix or matrix and particles. The single-pore volumes were normalized by the volume of a large particle $\left(V_{\text {por }} / \pi{\overline{r_{L}}}^{2}\right)$ since the parameter $r_{L}$ was kept constant between all samples. It was previously shown that an increase of particle-size ratio $\gamma$ results in a higher particle volume fraction $\rho_{p}$, and therefore less overall pore space. This does not, however, reveal any information on the distribution of the individual pore sizes in the sample. It has been shown that the spatial configuration of voids in granular samples are crucial for the mechanical behavior [30]. Parameters such as void radius, void orientation, and distribution in the sample affect the mechanical response [14-16].

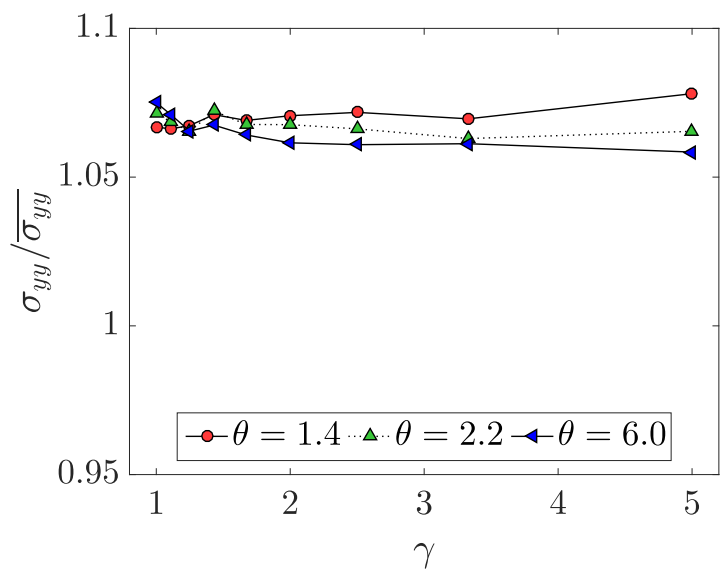

(a)

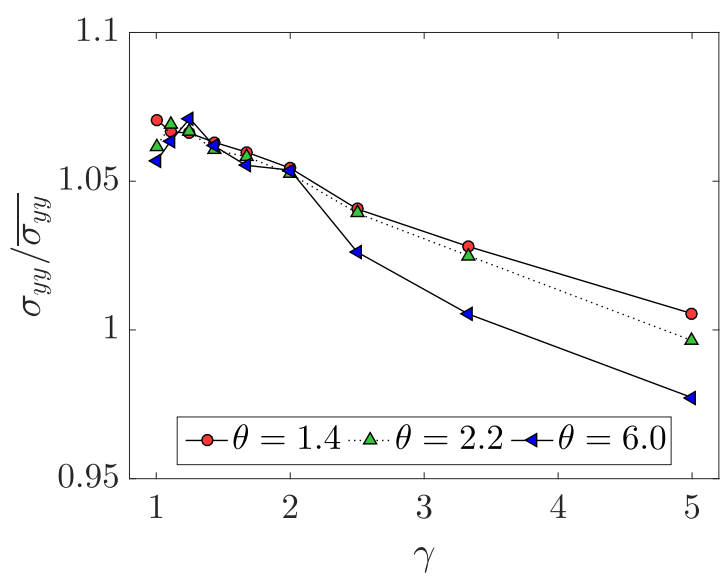

(b)

FIG. 11. The mean stress in full samples in (a) large particles and (b) small particles.

The pore volume defines the maximum void volume and is therefore an important parameter.

Several examples of probability density functions (PDF) of the single-pore volumes are shown in Fig. 6(a) for $\theta=1.4$ and different values of $\gamma$. The single-pore volumes are distributed over a broad range of values with a peak value centered on an increasingly large value of pore volume for decreasing $\gamma$. The peak value decreases as $\gamma$ becomes larger. This means that the samples are more homogeneous in pore distribution for higher values of $\gamma$. Figure 6(b) shows that the mean value of the normalized single-pore volume is a nearly linear as a function of $1 / \gamma$, which is proportional to the size of small particles. The small variability in this dependence is controlled by the volume ratio.

The standard deviation of the normalized single-pore volumes is rather large, as shown in Fig. 6(c), and increases with $1 / \gamma$ in the same manner as the mean of single-pore volume. This indicates inherent inhomogeneities of the packings due to the existence of both densely and loosely packed zones (see Fig. 3, for example). Laubie et al. [30] used the standard variation of local porosity as a parameter for the disorder of pore spaces. Even though not calculated in the same way, the 


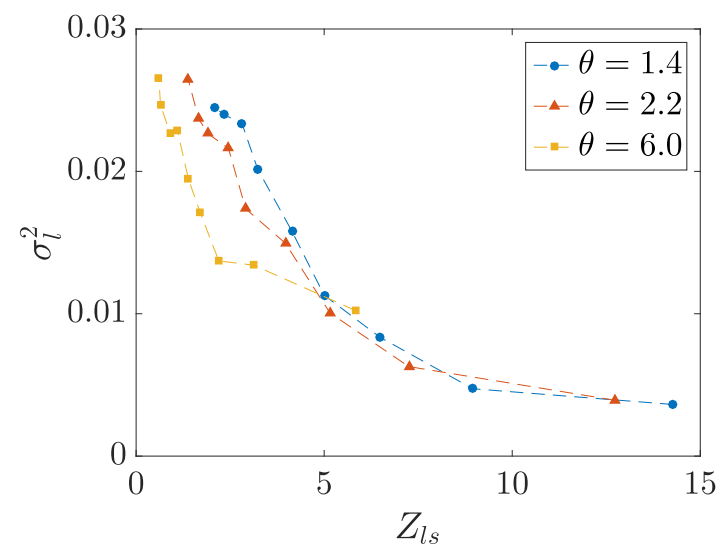

(a)

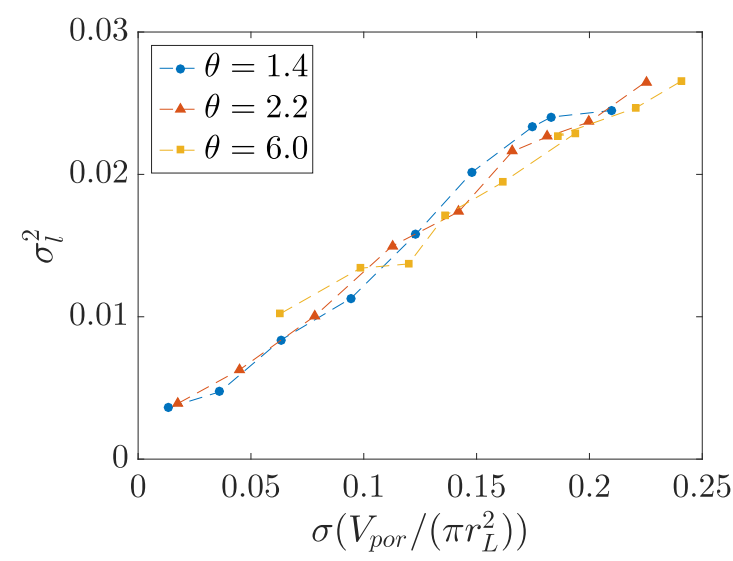

(b)

FIG. 12. Variance $\sigma_{l}^{2}$ of stress PDFs of large particles in full samples (no voids) versus (a) partial coordination number $Z_{l s}$, and (b) standard deviation of single-pore volume, for selected values of $\theta$.

standard deviation of single-pore volumes may be considered in a similar fashion as disorder parameter.

As mentioned previously, the void volumes, orientations and spatial distributions have a significant effect on stress transmission and other mechanical properties of a granular material [14-16,30]. The void volumes depend on the amount of matrix, filling method, and initially available pore space.

We saw that the size of the interstitial space between particles directly depends on that of small particles $r_{s} \propto 1 / \gamma$. Therefore, due to the specific filling method used in our study (bridges between particles within a certain distance), the void size is expected to depend also strongly on the particle-size distribution. For the interstitial spaces, a decrease in the small particle size results in a decrease in the average single-pore size. This is also true for the average single-void size shown in Fig. 7(a). Obviously, the addition of any amount of matrix into the pore spaces decreases the mean single-void volume.

The relation between the amount of matrix and the normalized mean single-void volume, shown in Fig. 7(b), is however less intuitive. The matrix filling ratio $S_{m}=\frac{\rho_{m}}{1-\rho_{p}}$ quantifies the fraction of nonparticle space filled with matrix; $S_{m}=0$ corresponds to a sample with no matrix and $S_{m}=1$ to a sample where all interstitial spaces are filled with matrix. Generally, a decrease of the mean single-void volume with increasing $S_{m}$ is observed [Fig. 7(b)], with the mean single-void volume being lowest for the highest values of matrix filling ratio. A striking exception to this is that in all partially filled samples the mean void volume is highest for the intermediate values $0.4<S_{m}<$ 0.7 , and not, as might be expected, for the lowest values of $S_{m}$. To understand this, let us consider the PDFs of the void volumes in Fig. 8. We see that for $S_{m}<0.5$, a dominant peak at small void volumes is present, while larger void volumes are also present. The first peak shifts to smaller values as $S_{m}$ increases from 0 to $S_{m}<0.4$. This shows that the addition of more matrix reduces the void volumes, certainly due to the specific matrix filling method used. However, once $S_{m}$ is larger than a threshold value $\approx 0.4$, the peak at very small void volumes shrinks and disappears. This indicates that beyond this value the very small voids disappear, meaning that the corresponding pores are completely filled with matrix. Due to the lack of very small voids, the mean single void volume suddenly increases. Beyond this threshold, the addition of matrix continues to reduce the volume of the remaining voids again, as observed by the shift of the highest peak toward smaller values.

Interestingly, this behavior has a significant effect on the coefficient of variation $(\mathrm{CV})$ of the void volume shown in Fig. 7(d). At low values of $S_{m}$ up to a threshold of about 0.4 , the CV of single-void volume increases. This is most likely caused by the size reduction of the very small voids while at the same time the larger voids are not affected by the addition of matrix. The particles surrounding the largest voids are too far away from each other to be connected by a matrix bridge. This behavior is clearly an effect of the matrix filling method. As the threshold value $S_{m} \approx 0.4$ is reached, a sudden decrease in $\mathrm{CV}$ down to values $<1$ is observed, indicating that the void volumes of these medium filled samples are much more homogeneous. Then again, as $S_{m}$ increases further, the $\mathrm{CV}$ increases too. The influence of the particle-size ratio $\gamma$ seems to be limited to its effect on the available pore volumes and therefore on the maximum void volume. In similar ranges of $S_{m}$, both higher and lower values of $\gamma$ can lead to the same value of $\mathrm{CV}$.

\section{STRESS TRANSMISSION}

\section{A. Stresses in full samples}

The stress fields in all phases of all samples were calculated under tensile loading by means of the peridynamic method. Figure 9(a) displays the probability density functions (PDF) of vertical stresses normalized by the mean stress in all phases in a filled sample $\left(\rho_{m}+\rho_{p}=1, \rho_{v}=0\right)$. The PDFs reflect the vertical stress values at the scale of the spatial resolution of the peridynamic calculations. We see that the largest stresses extend to two times the mean stress, but the ratio between the largest and smallest stresses can be much higher. This inhomogeneity arises from stress concentration due to pores, stress chains along contiguous particles and arching effect that tends to screen parts of the system. The statistics has not exactly the same precision for all samples since the same spatial resolution is used for all samples irrespective of the 


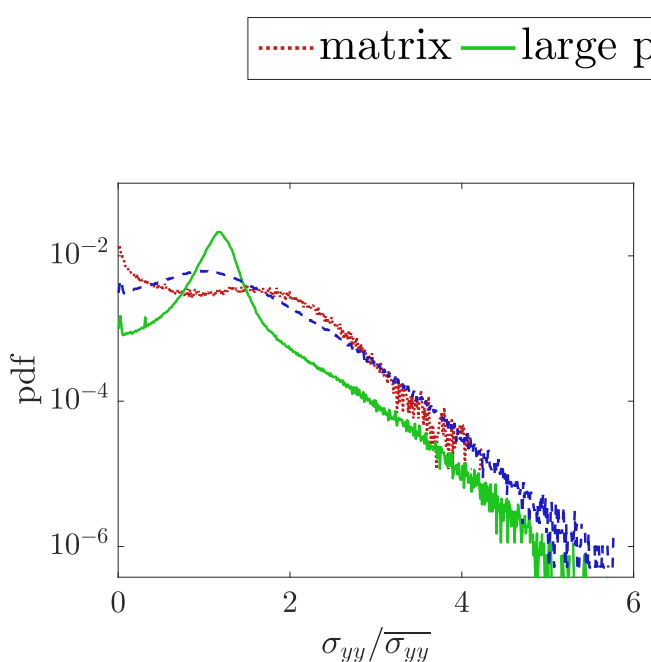

(a)

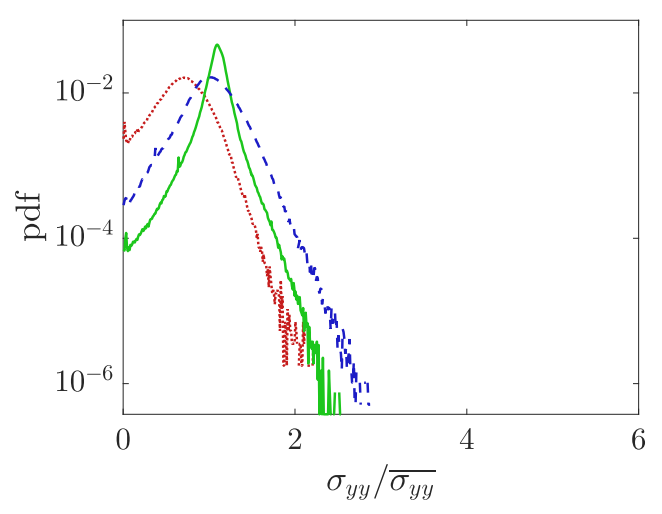

(c)

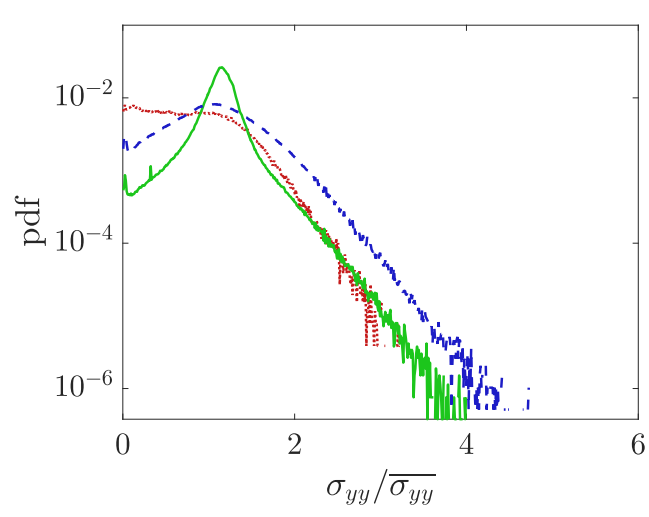

(b)

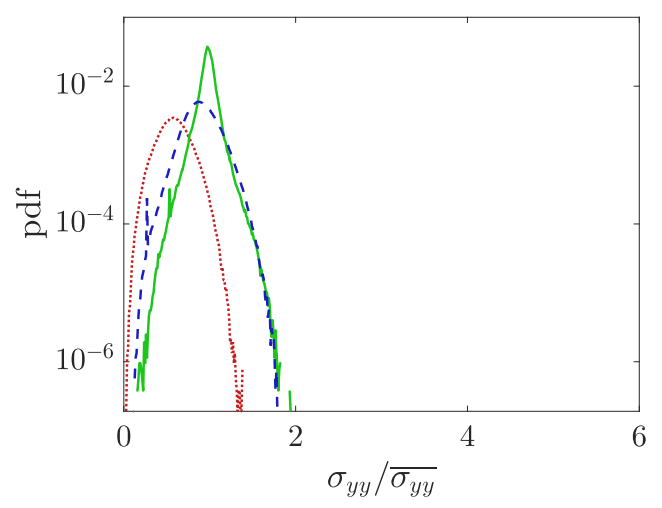

(d)

FIG. 13. The normalized vertical stress distributions in different phases for different amounts of matrix volume fraction for $\gamma=5$ and $\theta=1.32$ : (a) very low $S_{m}$, (b) medium $S_{m}$, (c) high $S_{m}$, and (d) completely full.

value of $\gamma$. Hence, the precision for extreme values of the stress is low in samples with $\gamma=5$. In those samples, the edges of small particles are not smooth enough to render well-defined values. In particular, we observe a few spikes on the distributions on Fig. 9(a), echoing such edge effects. Nevertheless, up to such minor effects and even by keeping the full range of stress values, all plots are smooth enough to reflect the effects of the underlying microstructure on stress transmission.

The stress PDFs show that the matrix carries lower stresses whereas the small and large particles carry higher stresses. This is clearly a consequence of the lower value of the matrix Young modulus. The PDF in large particles is narrower than that in small particles, and it shows a pronounced peak around the mean stress. The PDFs in different material phases and for different values of $\gamma$ are shown in Figs. 9(b)-9(d). An increase in particle-size ratio leads to increasingly pronounced stress peak. However, the phases are affected differently. The stress distribution in the matrix gets slightly narrower with increasing $\gamma$ as seen in Fig. 9(d). For the small particle phase a slight shift toward lower stress values is observed with increasing $\gamma$; see Fig. 9(c). The particle-size ratio has the most significant effect on the stresses in large particles; see Fig. 9(b). The higher the size ratio $\gamma$, the more homogeneously the stresses are distributed in large particles, resulting in a higher PDF peak. This behavior can be qualitatively understood from the observations in the previous section on the partial coordination number of large particles $Z_{l}$, which constantly increases with $\gamma$, and implies a more homogeneous distribution of stresses within the large particles as illustrated in Fig. 10 for a particle having a small number of contacts and a particle with large number of contacts.

In Fig. 11 we have plotted separately the mean stress in the classes of large and small particles as a function of $\gamma$ for three different values of $\theta$. Interestingly, the mean stress in large particles is nearly independent of $\gamma$ and slightly (about 7\%) above that in the whole sample. However, the mean stress of small particles declines as $\gamma$ increases and tends to a value below the mean stress of the whole sample. This is consistent with the shift of the PDF's for small particles toward lower stress values with increasing $\gamma$ as observed in Fig. 9(c). This trend is the same for all values of $\theta$, and it implies that an increasingly higher fraction of stress is transferred from small particles to the matrix. This means that the matrix plays a more active role in stress transmission when its effect is mediated by the small particles.

To further characterize the effect of particle sizes on stress transmission in large particles, we calculated the variance $\sigma_{l}^{2}$ 


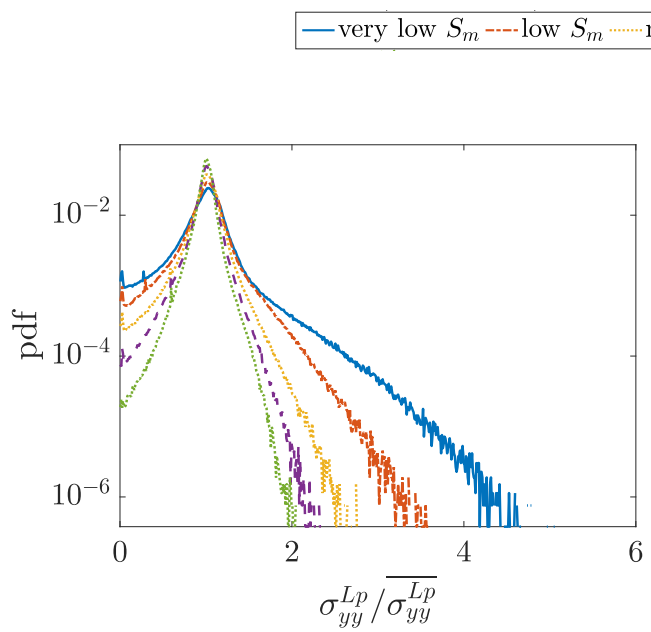

(a)

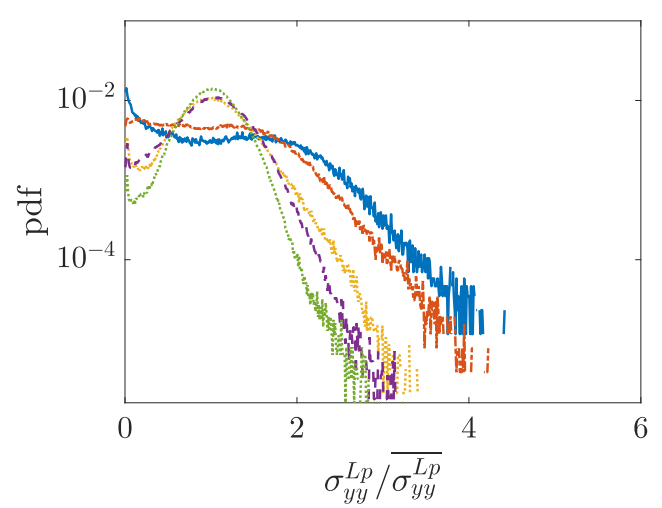

(c)

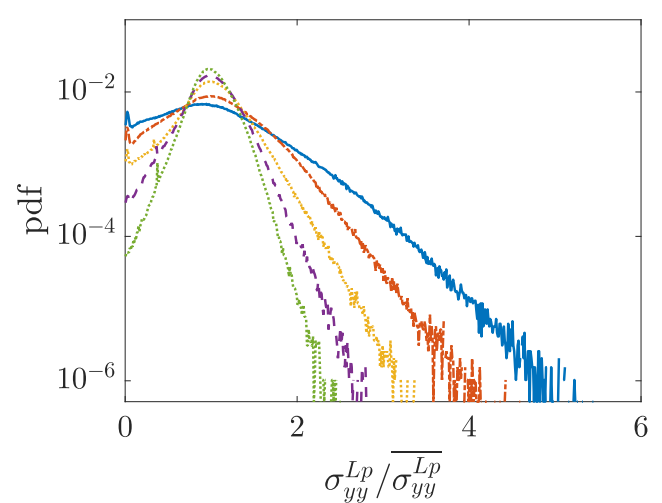

(b)

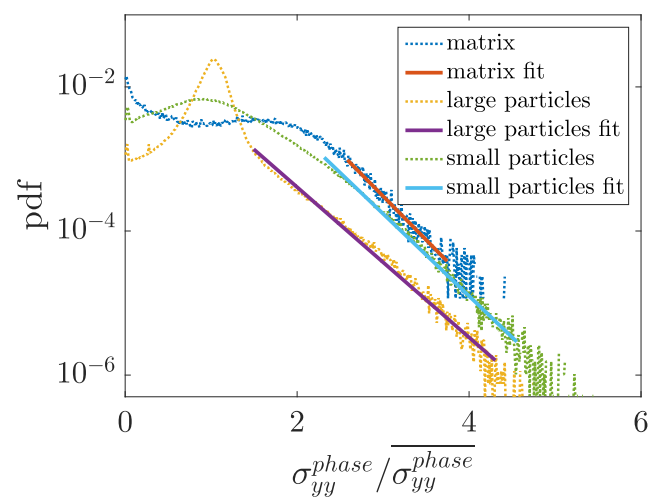

(d)

FIG. 14. Stress distributions normalised for each material phase separately $\sigma_{y y}^{\text {phase }} / \overline{\sigma_{y y}^{\text {phase }}}$ with changing matrix saturation for $\gamma=5.00$ and $\theta=1.32$ in (a) large particles, (b) small particles, and (c) matrix. Panel (d) shows examples of the exponential fits performed on the distribution tails.

of stresses. The variance reflects the degree of inhomogeneity of the stresses. Figure 12(a) shows that the variance for large particle stresses $\sigma_{l}^{2}$ declines rapidly with increasing partial coordination number $Z_{l s}$. More interestingly, Fig. 12(b) shows that $\sigma_{l}^{2}$ increases with the standard deviation of singlepore volumes. This relation between the standard deviation of single-pore volume and the variance of stresses in large particles is linear and independent of $\theta$. Previously, we argued that the standard deviation of the distribution of single-pore volumes may be interpreted as an indicator of disorder in the sample. This argument is now supported by the strong correlation between this standard deviation of pore volumes and stress transmission in large particles. The standard deviation of single-pore volumes is the best indicator of the variability of stresses in large particles. The stresses are therefore more homogeneously distributed in samples with pores more homogeneously distributed and in samples with more contact points around large particles. This happens at larger particle-size ratio $\gamma$ and/or a higher volume fraction of small particles (lower $\theta$ ); see Figs. 5(f) and 6(c).

The shapes of the PDFs in Fig. 9(b) cannot be fully and exactly fitted by Gaussian functions. The behavior of the statistics around the mean stress would be more adequately fitted by a q-Gaussian distribution (not shown) [31]. The
q-Gaussian distribution was previously used by Combe et al. to analyze the broad distributions of particle displacements in sheared granular materials [32]. Our data indicate that the value of the exponent $q$ is an increasing function of $\gamma$, implying the non-Gaussian broadening of the stress distribution with size ratio. These features of stress distribution functions will be analyzed in more detail in an upcoming paper.

\section{B. Stresses in partially filled samples}

The range of the stress values in partly cemented samples, as shown in Figs. 13(a)-13(c) is significantly larger than in full samples as observed in Fig. 13(d). In contrast to full samples, the highest stresses are not shared between small and large particles, but they are mostly carried by small particles. Moreover, the lesser a sample contains matrix, the more this matrix is involved in carrying stresses above the average stress.

Figure 14 shows the evolution of the stress PDFs in the different phases with increasing matrix saturation $S_{m}$. The stress distributions are normalized separately for each phase. All the PDFs are for the highest particle-size ratio $\gamma=5.00$. It is observed that the values of the highest stresses in each phase decrease with increasing matrix filling. With increasing matrix 
$\circ \gamma=5.00 \cdots \gamma=3.33-\square=2.50-\Delta \gamma=2.00 \multimap \gamma=1.67$ $\times \gamma=1.43-\triangleleft \gamma=1.25 \rightarrow \gamma=1.11 \multimap \gamma=1.00$

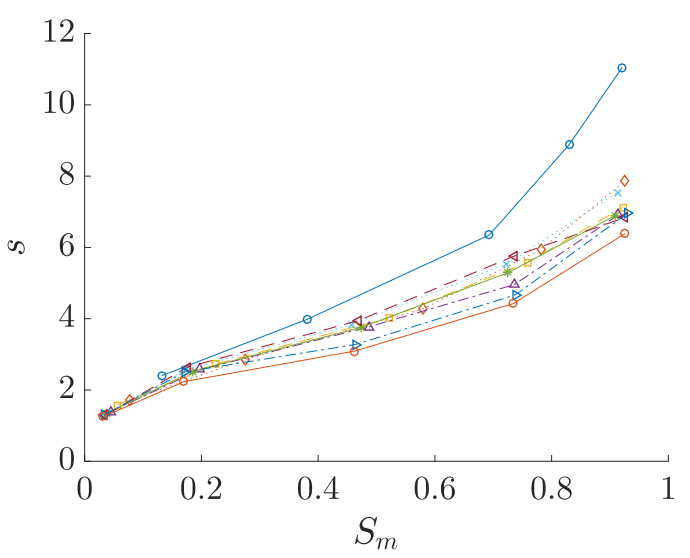

(a)

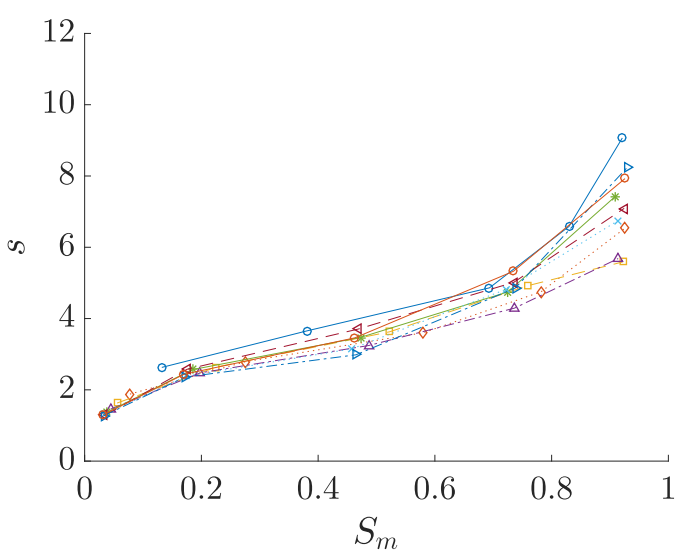

(b)

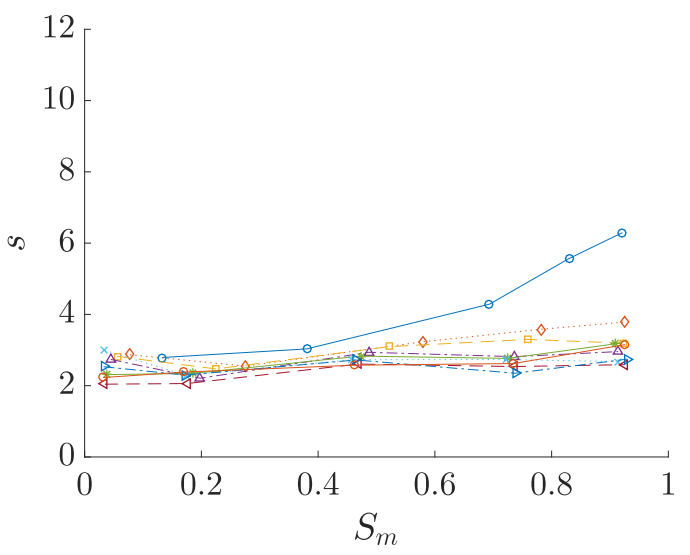

(c)

FIG. 15. The slope of the exponential decay of the tail of stress PDFs in (a) large particles, (b) small particles, and (c) matrix versus the matrix saturation $S_{m}$.

filling, the stresses in all phases also become more homogeneous. The center of the PDF maintains its nearly Gaussian shape while a marked exponential tail develops for lower $S_{m}$. The stress distributions in small particles [Fig. 14(b)] are broader than in large particles, as we already observed in the case of full samples. For small particles, the exponential tail also becomes more prominent with decreasing matrix content. The stress distributions in the matrix phase [Fig. 14(c)] undergo the greatest qualitative change between low and medium matrix content. While the PDFs for medium, high, and very high $S_{m}$ are similar, at low and very low matrix content, the distributions are wider, without a clear peak and with a high number of very low stresses.

Very high stresses and the corresponding exponential falloffs are important for the mechanical behavior of a cemented granular material. We previously showed that the highest stresses occur at the particle contact points. In the matrix filling method used in this work, the increase in the amount of matrix is achieved by an increase in the matrix bridge width between particles. Therefore less amount of matrix implies narrower cementation regions between particles, and thus a higher concentration of stresses.

The exponential decay of stresses can be used to quantify the degree of stress inhomogeneity in different phases. By fitting the PDF in the range of high stresses to a decreasing exponential form $p d f=\left[\frac{\sigma_{y y}}{\bar{\sigma}_{y y}}\right]_{0} e^{\left[-s \cdot\left(\frac{\sigma_{y y}}{\sigma_{y y}}\right)\right]}$, as shown in Fig. 14(d), we obtain the exponent $s$, which is all the more small that the stress distribution is more inhomogeneous. Figures 15(a) and 15(b) show $s$ in samples with $\theta=1.32$ and different values of $\gamma$ as a function of matrix volume fraction $S_{m}$. We see that $s$ increases in large and small particles with increasing matrix saturation. The particle-size ratio $\gamma$ has less influence than the amount of matrix for low values of $S_{m}$, but becomes more influent for higher $S_{m}$. This effect is more pronounced in large particles for $\gamma=5.00$.

Laubie et al. [30] used the excess kurtosis of stress PDFs in porous materials as a descriptor of the broadening of stress distributions. They found that the excess kurtosis grows quadratically with a disorder parameter calculated from the local porosity. We calculated the excess kurtosis in our different samples and in different phases. But the results were more difficult to interpret. For example, the value of the excess kurtosis in large particles was found to have its highest value for the largest value of $\gamma$ (not shown) despite the aforementioned observation that larger values of $\gamma$ lead to more homogeneous stress distributions. This is also the case for partially filled samples (graphs not shown). This discrepancy can be explained by the fact that our distributions are not symmetric around the mean stress. In particular, the occurrence of very small stresses can be significant.

\section{CONCLUSION}

In this work, we showed the complex interplay between particle-size distribution and matrix volume fraction for stress transmission in bidisperse granular materials, representing the simplest polydisperse system governed by only two parameters: size ratio and relative proportions of large and small particles. When the size ratio is high, the small particles act as a pore-filling material with respect to large particles if their proportion is high. The mixture can then be thought of as an assembly of large particles inside a matrix of small particles. At lower size ratios, the small particles play a structural role, 
and together with large particles they control the properties of the texture and force transmission. Therefore, the effect of added cementing matrix on stress transmission depends closely on the texture. For example, at high proportion of small particles and high size ratio, the effect of the cementing matrix on stress transmission and cohesion of the material is amplified by the phase of small particles filling the space between large particles.

In this paper, we quantified these features by systematic simulations using the peridynamic method for a broad range of particle-size ratios, volume fractions of large and small particles, and the amount of the cementing matrix. This is the first time the peridynamic method is applied to investigate cemented granular materials. It has the advantage of providing access to stresses inside the particles and matrix. As expected, the most homogeneous stress distribution occurs for high size ratio and large volume fraction of small particles. The tensile stresses show non-Gaussian distributions with a width that increases with increasing porosity. At higher porosity, a decreasing exponential distribution of strong tensile stresses develops and replaces the Gaussian distribution. This exponential fall-off of the probability density of strong stresses is a hall-mark of granular materials, reflecting granular disorder. One important difference is that in the case of granular materials the exponential distribution concerns contact forces whereas we have here a similar behavior for stresses everywhere in the system, inside both the particles and the matrix. The exponential fall-off is a signature of the presence of "stress chains," i.e., nearly aligned sequences of particles transmitting strong forces. The force chains are more commonly known for cohesionless granular materials under compressive confining pressure. However, similar chains of tensile forces or stresses occur under tensile stress in both cemented granular materials, as in our system, and in wet granular materials [33-35]. The tensile force chains are characterized by an exponential probability density distribution as the compressive force chains. This local concentration of stresses is not directly related to porosity but reflect rather the contact network. The exponential part of the distribution broadens with decreasing matrix volume fraction and particlesize ratio, indicating higher structural disorder. The tensile stress and force chains were previously studied for cemented and wet granular materials.

Our results show that the role of porosity for stress transmission is not as straightforward as in "unstructured" porous materials, i.e., a simple distribution of pores in a matrix. In granular materials, the distribution of pores reflects the structure of the contact network. Our results clearly show that stress inhomogeneity in full samples is controlled by the standard deviation of pore-size distribution rather than porosity. In general, the particle-size distribution controls the stress transmission properties at low void volumes (high matrix volume fraction).

This work can be extended along different directions:

(1) In this work, the matrix phase was distributed almost homogeneously according to a well-defined protocol. More work is necessary to consider more general distributions of particle sizes and cementing matrix.

(2) The mechanical parameters $K_{p}, K_{m}$, and $K_{p m}$ were fixed to their values for wheat endosperm obtained by means of AFM and nanoindentation experiments while using a broad range of values of $\gamma$ and $\theta$. Hence, the results can be more specifically used for the prediction of the texture and stress transmission in wheat endosperm for different values of $\gamma$ and $\theta$, which can be genetically modified. Results on the effect of the mechanical parameters can be found in Refs. [1-3].

(3) The 2D setting used in this paper made it possible to perform extensive parametric studies. But it is obvious that an extension to 3D simulations can provide a more realistic description of stress transmission.

(4) Finally, a similar investigation can be performed under different loading conditions, e.g., under shear stress or compressive stress increments. The role of contacts between particles becomes more prominent in compression and a larger amount of stress will be transmitted through the particle phase.

(5) The stress transmission as discussed in this paper provides a general picture of the internal state of cemented binary granular materials. In an upcoming paper, we will be interested in the effect of the texture on the elastic moduli of these materials.

\section{ACKNOWLEDGMENTS}

We thank INRAE (division for Science for Food and Bioproduct Engineering, Grant No. 140948) and Labex Numev (Grant No. 2014-1-012) for the Ph.D. grant for K. Heinze.

\section{APPENDIX: SAMPLE CONSTRUCTION USING FAST POISSON DISC SAMPLING}

Poisson disk sampling is used in many computer graphics applications. It is a geometrical approach which consists in generating a collection of points (samples) that are at smallest possible distance from one another. This specific spatial distribution is considered ideal for image rendering [36].

The fast Poisson disk sampling (FPDS) algorithm used in this paper was adapted from the note of Brisdon [17] for the case of bidisperse samples. Although the method is not capable to produce mechanically stable samples, it is used as a first step to pack particles into dense samples. One major feature is its ability to generate dense samples with a homogeneous distribution of big particles with respect to the small ones. This point is important to limit segregation effects prior to dynamic compaction using DEM.

In this algorithm we assume $N$ to be the total number of particles. For each particle $i$, the radii $r_{i}$ are chosen according to a truncated Gaussian distribution in the range $r_{i} \in$ $\left[r_{\min }, r_{\max }\right]$. We consider a list of so-called "active particles" that are particles around which there is enough free space to place a new particle in contact with them. A background grid is created to ease the spatial research of particles. This grid is similar to the a classical Verlet grid [37], but each cell is supposed to contain at most one particle center. Moreover, the cell size is bounded by $r_{\min } / \sqrt{N}$ in $2 \mathrm{D}$ so that the grid can be implemented as a $\mathrm{N}$-dimensional array containing the particle indices. Before running the algorithm, an initial seed particle is placed in the domain and the its index is added to the active list.

(1) While the active list is not empty, randomly select a particle $j$. 
(a) Randomly draw a radius $r_{k}$ and an angular position of the center of the particle $k$ under the constraint that the distance $d_{j k}$ between the two centers is in the range $\left[r_{j}+\right.$ $r_{k}, r_{j}+r_{k}+\delta$ ], where $\delta$ is the gap between particles. Its value can be set to 0 to have particles in contact or with any arbitrary or random positive value.

(b) Using the grid to test the neighboring particles, check if $k$ is at a distance greater than $\delta$ of other particles.

(c) If this is the case, then add the particle to the sample and the index $k$ to the active list. Otherwise, repeat this loop, and after a maximum number of trials, remove $j$ from the active list.
Any walls or periodic boundary conditions can easily be taken into account by incorporating additional geometric constraints. In this case, the simulation will last until the domain is fully filled with particles.

In our simulations, the big and small particles are packed with the same algorithm in two steps:

(1) In the first step, the big particles are placed by letting enough space between them using the $\delta$ parameter.

(2) In the second step, the small particles are placed in the remaining free space between the bigger discs by setting $\delta=0$.
[1] V. Topin, J.-Y. Delenne, F. Radjaï, L. Brendel, and F. Mabille, Eur. Phys. J. E 23, 413 (2007).

[2] V. Topin, F. Radjai, J.-Y. Delenne, A. Sadoudi, and F. Mabille, J. Cereal Sci. 47, 347 (2008).

[3] E. Chichti, V. Lullien-Pellerin, M. George, F. Radjai, R. Affès, and J.-Y. Delenne, J. Food Eng. 190, 40 (2016).

[4] C. Chang, M. D. Zoback, and A. Khaksar, J. Pet. Sci. Eng. 51, 223 (2006).

[5] R. Holtzman, Int. J. Numer. Anal. Methods Geomech. 36, 944 (2012).

[6] O. Buyukozturk and B. Hearing, Int. J. Solids Struct. 35, 4055 (1998).

[7] T. Ueda, T. Matsushima, and Y. Yamada, Granular Matter 13, 731 (2011).

[8] J. Wiącek and M. Molenda, Particuology 16, 91 (2014).

[9] J. Wiącek, Granular Matter 18, 42 (2016).

[10] J. Wiącek, M. Stasiak, and P. Parafiniuk, Arch. Civil Mech. Eng. 17, 271 (2017).

[11] J. Peng, L. N. Y. Wong, and C. I. Teh, Int. J. Rock Mech. Min. Sciences 100, 207 (2017).

[12] C. Voivret, F. Radjai, J.-Y. Delenne, and M. S. El Youssoufi, Phys. Rev. E 76, 021301 (2007).

[13] C. Voivret, F. Radjai, J.-Y. Delenne, and M. S. El Youssoufi, Phys. Rev. Lett. 102, 178001 (2009).

[14] P. Baud, T. fong Wong, and W. Zhu, Int. J. Rock Mech. Min. Sciences 67, 202 (2014).

[15] L. Griffiths, M. J. Heap, T. Xu, C. feng Chen, and P. Baud, J. Struct. Geol. 96, 149 (2017).

[16] A. Bubeck, R. Walker, D. Healy, M. Dobbs, and D. Holwell, Earth Planet. Sci. Lett. 457, 38 (2017).

[17] R. Bridson, ACM SIGGRAPH 2007 Sketches (ACM, New York, NY, 2007).
[18] S. Torbert, Applied Computer Science (Springer, Berlin, 2016).

[19] V. Richefeu, F. Radjai, and J.-Y. Delenne, Comput. Geotech. 80, 353 (2016).

[20] S. Silling and E. Askari, Comput. Struct. 83, 1526 (2005).

[21] X. Frank, J.-Y. Delenne, and F. Radjai, EPJ Web Conf. 140, 02029 (2017).

[22] Y. D. Ha and F. Bobaru, Eng. Fract. Mech. 78, 1156 (2011).

[23] E. Chichti, M. George, J.-Y. Delenne, F. Radjai, and V. LullienPellerin, Eur. Polymer J. 49, 3788 (2013).

[24] E. Chichti, M. George, J.-Y. Delenne, and V. Lullien-Pellerin, Plant Sci. 239, 1 (2015).

[25] R. K. McGeary, J. Am. Ceram. Soc. 44, 513 (1961).

[26] J. Dodds, J. Colloid Interface Sci. 77, 317 (1980).

[27] P. V. Lade, C. D. Liggio, and J. A. Yamamuro, Geotech. Test. J. 21, 336 (1998).

[28] R. P. Dias, J. A. Teixeira, M. G. Mota, and A. I. Yelshin, Ind. Eng. Chem. Res. 43, 7912 (2004).

[29] L. Meng, P. Lu, and S. Li, Particuology 16, 155 (2014).

[30] H. Laubie, F. Radjai, R. Pellenq, and F.-J. Ulm, Phys. Rev. Lett. 119, 075501 (2017).

[31] C. Tsallis, J. Stat. Phys. 52, 479 (1988).

[32] G. Combe, V. Richefeu, M. Stasiak, and A. P. F. Atman, Phys. Rev. Lett. 115, 238301 (2015).

[33] V. Topin, F. Radjaï, J.-Y. Delenne, and F. Mabille, Powder Technol. 190, 215 (2009).

[34] V. Richefeu, F. Radjaï, and M. S. El Youssoufi, Eur. Phys. J. E 21, 359 (2007)

[35] V. Richefeu, M. S. El Youssoufi, E. Azéma, and F. Radjaï, Powder Technol. 190, 258 (2009).

[36] R. L. Cook, ACM Trans. Graph. 5, 51 (1986).

[37] M. Allen and D. Tildesley, Computer Simulation of Liquids (Clarendon Press, Oxford, UK, 1987). 